\title{
Can Appropriate Diagnosis and Treatment of Childhood Asthma Reduce Excessive Antibiotic Usage?
}

\author{
Ahmet Hakan Gedik ${ }^{\mathrm{a}}$ Erkan Cakir $^{\mathrm{a}}$ Emin Ozkaya $^{\mathrm{b}}$ Engin Ari ${ }^{\mathrm{c}}$ \\ Mustafa Nursoy ${ }^{\text {b }}$ \\ Divisions of a Pediatric Pulmonology and ${ }^{b}$ Pediatric Allergy, and ${ }^{c}$ Department of Pediatrics, Faculty of Medicine, \\ Bezmialem Vakif University, Istanbul, Turkey
}

\section{Key Words}

Childhood $\cdot$ Asthma $\cdot$ Antibiotic usage

\begin{abstract}
Introduction: This study compared the frequency of antibiotic usage and the number of asthma episodes before and after the diagnosis and treatment of pediatric asthma patients who were followed up by specialists. Subjects and Methods: Included in this study were 334 patients (211 males and 123 females) of 2-16 years of age who were diagnosed with asthma and followed up for at least 1 year in our clinic. The frequency of antibiotic usage and the number of asthma episodes in the year prior to diagnosis and treatment were compared to these same variables after 1 year of follow-up by specialists. Results: The median age was 84 months (range: 24-192) and 212 (63\%) children were at school or in day care centers. Atopy and a family history of asthma were present in $200(60 \%)$ of the patients, and 137 (41\%) reported that at least one member of their household smoked. Antibiotics were used a median number of 7 times [interquartile range $(\mathrm{IQR})=6$ ] in the year before the asthma diagnosis, and 2 times $(I Q R=3)$ during the year after treatment $(p<0.001)$. The mean number of asthma episodes before diagnosis, i.e. $4(\mathrm{IQR}=8)$ was reduced to $0(\mathrm{IQR}=2)$ in
\end{abstract}

the year after treatment when the patients were followed up by specialists $(p<0.001)$. Conclusion: This study shows that appropriate diagnosis and treatment of childhood asthma significantly reduce the frequency of antibiotic usage and the number of asthmatic episodes. (c) 2014 S. Karger AG, Basel

\section{Introduction}

Undiagnosed asthmatic patients who present with cough, wheezing, shortness of breath and other common respiratory symptoms are sometimes mistakenly diagnosed with upper or lower respiratory tract infections [1]. In children with asthma-like symptoms, such as recurrent episodes of wheezing, cough and shortness of breath, it has been reported that treatments more often consist of antibiotics and cough medicines than of asthma drugs [2]. Contrary to the recommendations in the international guidelines, antibiotics are often prescribed instead of drugs to treat asthma [1]. Excessive antibiotic usage by asthmatic patients has been reported in the literature [15]. A doctor's education, training and experience can result in an appropriate diagnosis, which in turn can reduce antibiotic usage by asthmatics [6]. Knowledge of appro-

\begin{tabular}{ll}
\hline KARGER & $\begin{array}{l}\text { (c) 2014 S. Karger AG, Basel } \\
\text { 1011-7571/14/0235-0443\$39.50/0 Open access }\end{array}$ \\
$\begin{array}{l}\text { E-Mail karger@karger.com } \\
\text { www.karger.com/mpp }\end{array}$ & $\begin{array}{l}\text { This is an Open Access article licensed under the terms of the } \\
\text { Creative Commons Attribution-NonCommercial 3.0 Un- } \\
\text { ported license (CC BY-NC) (www.karger.com/OA-license), } \\
\text { applicable to the online version of the article only. Distribu- } \\
\text { tion permitted for non-commercial purposes only. }\end{array}$
\end{tabular}

Ahmet Hakan Gedik, MD

Division of Pediatric Pulmonology, Faculty of Medicine

Bezmialem Vakif University, Adnan Menderes Bulvarı (Vatan Cad.)

TR-34093 Fatih, Istanbul (Turkey)

E-Mail ahakangedik@hotmail.com 
priate medication as recommended in the guidelines could result in fewer prescriptions of antibiotics for asthma patients [1], and the education and regular follow-up of patients also improve asthma self-management. This study compared the frequency of antibiotic usage and the number of asthma episodes before and after the diagnosis and treatment of asthma.

\section{Subjects and Methods}

\section{Study Design and Patients}

Included in the study were 334 patients (2-16 years of age) who were diagnosed with asthma and admitted to the Divisions of Pediatric Allergy and Pediatric Pulmonology, Faculty of Medicine, Bezmialem Vakif University. Their demographic data and medical history for the year prior to diagnosis were obtained from parent reports at the first visit and retrospectively from a national database. After enrollment into the study, patients were prospectively followed up between September 2012 and September 2013. Those with immunodeficiency or on antibiotic prophylaxis for other diseases such as latent tuberculosis or repeating urinary tract infections were excluded. The study was approved by the Research Ethics Committee of the Bezmialem Vakif University. Signed informed consent forms were obtained from the families of all patients.

\section{Asthma Diagnosis}

The diagnosis of asthma was based on symptoms and medical history supported by laboratory findings. In patients younger than 5 years, the modified Asthma Predictive Index and the 2011 Global Initiative for Asthma (GINA) report were used [7, 8]. Patients were considered positive for asthma if they had a history of $\geq 4$ wheezing episodes in 1 year. Besides this primary threshold, they had to fulfill at least one major or two minor criteria. The major criteria included a parental history of asthma, physician-diagnosed atopic dermatitis and allergic sensitization to at least one aeroallergen. The minor criteria included wheezing unrelated to colds, peripheral blood eosinophils $(\geq 4 \%)$ and allergic sensitization to milk, eggs or peanuts. Skin prick tests, total immunoglobulin E levels, blood eosinophil counts and specific immunoglobulin E status (inhalant and food allergens) were used to evaluate the patients according to the criteria.

For patients older than 5 years, the GINA recommendations were used. Besides a family history of asthma or atopic disease, the suspicion of asthma increased in the presence of signs and symptoms which responded to antiasthma therapy [8]. Pulmonary function tests were used to measure airflow limitation and its reversibility. Skin prick tests, total immunoglobulin E levels, blood eosinophil counts and specific immunoglobulin E status (inhalant and food allergens) were also performed to establish an asthma diagnosis.

\section{Evaluation of the Patients}

At the first visit, simple questionnaires were filled out by a specialist, either a pediatric pulmonologist or an allergologist. These forms contained the demographic characteristics of the patients, their age at diagnosis, the parents' level of education, a history of smoking in the household, a history of atopy, the number of upper and lower airway infections (e.g. bronchitis and pneumonia) in the year prior to being diagnosed with asthma, the number of episodes of transient airway obstruction or respiratory distress and the frequency of antibiotic usage.

Turkish children younger than 18 years have government health insurance. In all health institutions including public and private hospitals as well as primary settings, children can be examined and given prescriptions free of charge. In addition, these prescriptions have to be entered into a database controlled by the Ministry of Health. It is against the law to buy antibiotics from pharmacies without digital prescriptions. The database also contains patients' International Statistical Classification of Disease and related problems (ICD) codes.

Data about wheezing, transient airway obstruction or respiratory distress episodes in the year prior to the diagnosis of asthma were obtained from the database. Antibiotic usage and respiratory infection data for this period were taken from the same database and confirmed by the parents at the first visit. The antibiotics used were obtained from digital prescriptions and parent reports; most common were amoxicillin-cluvulanate, cefuroxime-axetil, clarithromycin, ampicillin-sulbactam and azithromycin.

After diagnosis, patients were classified into 4 groups depending on their symptoms. Group 1 had mild and intermittent symptoms, group 2 had mild and persistent symptoms, group 3 had moderate and persistent symptoms and group 4 had severe symptoms. Patients received treatments based on the GINA recommendations [8]. The 80 (24\%) patients in group 1 received rapid-acting $\beta_{2}$-agonist as needed. The 184 (55\%) patients in group 2 were given low-dose inhaled corticosteroids (ICS). According to the severity of the symptoms, low-dose ICS plus a leukotriene modifier or low-dose ICS plus a long-acting $\beta_{2}$-agonist were used in the 53 $(16 \%)$ patients enrolled in group 3 . The 17 subjects in group 4 were given medium- or high-dose ICS plus a long-acting $\beta_{2}$-agonist. All patients were routinely evaluated every 3 months, during acute exacerbations and for other complaints.

At every visit, for each patient, the number of asthma episodes, antibiotic usage and the occurrence of respiratory infections were obtained from the parent reports and database and recorded. In addition, patients were educated about asthma and asthma medication, and an experienced nurse educated the patients about techniques for the inhalation of asthma drugs. At the end of 1 year, the frequency of antibiotic usage, the number of asthma episodes and upper or lower airway infections were again recorded.

\section{Statistical Analysis}

SPSS version 15.0 was used for analysis. The numerical parameters were described as the mean, median and standard deviation; distributions of the categorical measurements were determined by frequencies and percentages. The one-sample Kolmogorov-Smirnov test was used to evaluate the distributions of the number of episodes and frequency of antibiotic usage before and after diagnosis as well as during the treatment of asthma. As no normal distributions were found in either variable, the Wilcoxon signed-ranks test was used to compare differences between before and after diagnosis and during treatment with regard to the number of episodes and frequency of antibiotic use. Differences or change in the frequency of antibiotic use and the number of asthma episodes before and after the diagnosis and treatment of asthma were determined and written in two other columns in 
Table 1. Characteristics of the patients

\begin{tabular}{ll}
\hline Gender & \\
$\quad$ Male & $211(63)$ \\
$\quad$ Female & $123(37)$ \\
Median age (range), months & $84(24-192)$ \\
Mean age at diagnosis \pm SD, months & $61.40 \pm 33.29$ \\
Attendance at school or day care center & $212(63)$ \\
Household size & $4 \pm 1$ \\
At least one smoker in the household & $137(41)$ \\
Family history of doctor-diagnosed & \\
$\quad$ asthma and atopy & $200(60)$ \\
Inhaled-allergen positivity & $207(62)$ \\
High immunoglobulin E levels & $187(56)$ \\
Eosinophilia & $160(48)$ \\
Food allergies & $40(12)$ \\
Allergic rhinitis symptoms & $160(48)$ \\
Atopic dermatitis & $77(23)$
\end{tabular}

Unless otherwise indicated values represent $\mathrm{n}(\%)$.

the SPSS. The Spearmans correlation test and the Mann-Whitney $\mathrm{U}$ test showed associations between other factors such as gender, age at diagnosis, number of people in the household, parents' education levels, smoking status in the household, atopy history and any changes in the frequency of antibiotic usage or the number of asthma episodes. Multivariate linear regression analyses were used to evaluate the effect modification according to these factors. The multivariate analyses were adjusted for all factors simultaneously. $\mathrm{p}<0.05$ was considered to be statistically significant.

\section{Results}

The median age was 84 months (range 24-192) and the median age at diagnosis was 48 months (range $24-$ 156). Of the 334 children, $212(63 \%)$ were at school or in day care centers. Household size was $4 \pm 1$, and there was at least one smoker in the households of 137 (41\%) of the patients. A family history of doctor-diagnosed asthma and atopy was present in $200(60 \%)$ of the patients. The mothers of 20 patients $(6 \%)$ and the fathers of 33 patients (10\%) had graduated from university. Before study enrollment, all patients had had at least four wheezing episodes in their lives, 207 (62\%) had inhaledallergen positivity, 187 (56\%) had high immunoglobulin E levels and 160 (48\%) had eosinophilia. Allergic rhinitis symptoms were seen in $160(48 \%)$ patients, 77 (23\%) had atopic dermatitis and $40(12 \%)$ had food allergies. The patients were treated according to the GINA recommendations [8]; all took their drugs regu-

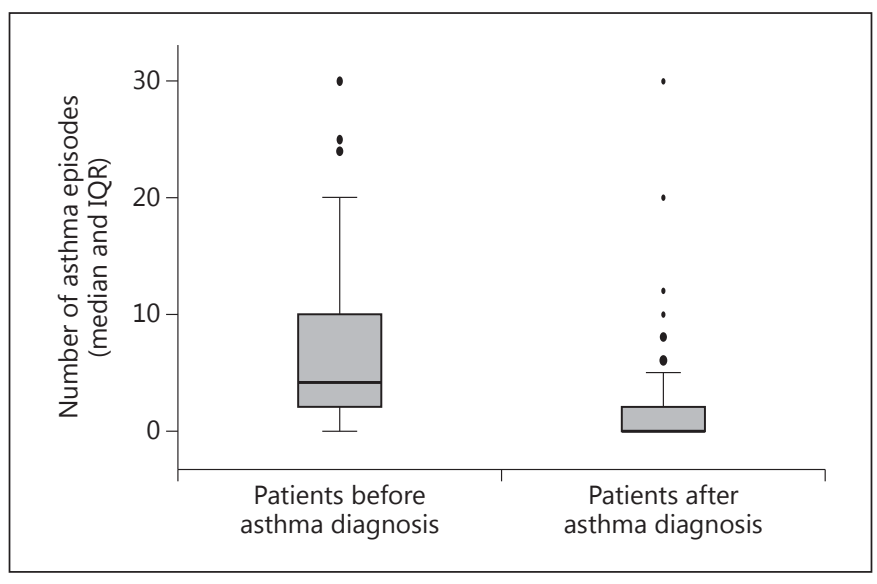

Fig. 1. Number of asthma episodes per patient per year before and after asthma diagnosis and treatment.

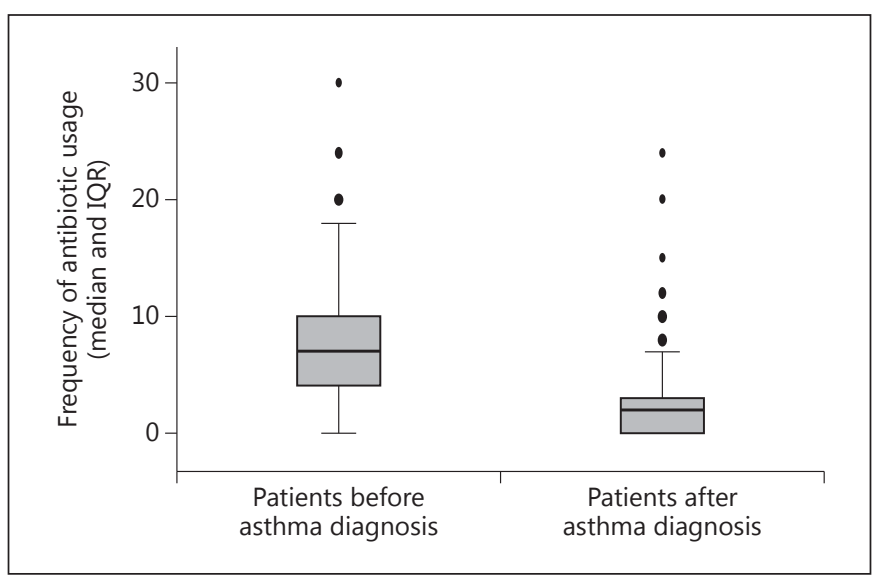

Fig. 2. Frequency of antibiotic usage by patient per year before and after asthma diagnosis and treatment.

larly and $301(90 \%)$ came to their evaluations every 3 months. The characteristics of the patients are shown in table 1.

For the year prior to diagnosis, 11 (3.2\%) parent reports about antibiotic usage and respiratory infections differed from what was recorded in the database. The number of times antibiotics were used differed by 1 in 9 of the patients and by 2 in 2 patients; such differences were due to the uncontrolled and spontaneous use of antibiotics prescribed at previous consultations. The agreement was $96.8 \%$ between the parent reports and the database for the year prior to the diagnosis of asthma. For the year after the diagnosis, there were no differences, i.e. the agreement was $100 \%$. 
Table 2. Associations between characteristics of patients and change in outcomes before and after the diagnosis

\begin{tabular}{lrrrrr}
\hline Factors & \multicolumn{2}{c}{ Asthma episode } & & \multicolumn{2}{c}{ Antibiotic use } \\
\cline { 2 - 3 } \cline { 6 - 7 } & beta & $\mathrm{p}$ & & beta & $\mathrm{p}$ \\
\hline Gender & 0.021 & 0.726 & & 0.061 & 0.321 \\
Diagnosis age & -0.119 & 0.051 & & -0.290 & 0.772 \\
Household number & 0.045 & 0.466 & & 0.035 & 0.582 \\
Mother's education & -0.038 & 0.551 & & -0.031 & 0.626 \\
$\begin{array}{l}\text { Father's education } \\
\text { Smoking in the }\end{array}$ & -0.039 & 0.538 & & -0.079 & 0.214 \\
$\quad$ household & 0.064 & 0.296 & & 0.007 & 0.915 \\
History of atopy & 0.042 & 0.489 & -0.035 & 0.559 \\
\hline
\end{tabular}

The median number of upper airway diseases before diagnosis of asthma was 5 [interquartile range $(\mathrm{IQR})=6$ ] per year which decreased to $1(\mathrm{IQR}=3)$ after treatment $(\mathrm{p}<0.001)$. The median number of lower airway diseases before diagnosis of $2(\mathrm{IQR}=5)$ per year dropped to 0 after treatment $(\mathrm{p}<0.001)$. The annual median number of asthma episodes before the asthma diagnosis was 4 (IQR $=8)$, decreasing to $0(\mathrm{IQR}=2)$ in the year after treatment ( $\mathrm{p}<0.001$; fig. 1). The median number of antibiotic administrations was $7(\mathrm{IQR}=6)$ per year before diagnosis and $2(\mathrm{IQR}=3)$ in the year following treatment $(\mathrm{p}<0.001$; fig. 2).

No statistically significant association was found between change in the frequency of antibiotic usage and number of asthma episodes before and after the diagnosis and gender, diagnosis age, number of household members, parents' education levels, household smoking status or history of atopy (table 2).

\section{Discussion}

This study showed that the frequency of antibiotic usage and the number of asthma episodes decreased to a statistically significant level after patients received appropriate treatment and regular follow-up by specialists.

The asthmatic children had more doctor visits, due to the fact that they are more symptomatic; this has been reported previously $[3,9]$. The possibility of misdiagnosing an acute asthma episode together with symptoms of increasing severity, like bronchiolitis, atypical pneumonia and other respiratory tract infections, means clinicians may choose to treat multiple possible etiologies [1]. Generally, $35 \%$ of asthmatics who experience episodes of re- spiratory tract infections are treated with antibiotics [10], even though these infections are most commonly viral in origin and trigger wheezing in young children [11]. Prior to admission to our clinic, the children had been frequently diagnosed with upper and lower airway infections, and so the use of antibiotics was high, which is similar to previous studies $[1,12]$. Reasons for using antibiotics include diagnostic uncertainty, the prophylaxis of secondary infections, an attempt to utilize the anti-inflammatory properties of macrolide antibiotics and the belief that unknown or noncultivable bacteria may be important in some asthmatic patients [1, 12]. Marra et al. [6] demonstrated that children eventually diagnosed with asthma had a higher rate of antibiotic usage than those who had never been diagnosed with asthma. In contrast to the outcomes of routine examinations, international guidelines specify that antibiotics should not be used for chronic asthma therapy or for acute exacerbations [8]. After enrollment, patients experiencing exacerbations were examined without delay as well as at regular follow-up appointments. If no evidence of bacterial infection was detected, we did not use antibiotics; exacerbations were treated with asthma drugs.

Numerous studies have assessed the role of antibiotics as a part of asthma therapy [13, 14]. De Boeck et al. [3] mentioned the strong tendency for health care providers to coprescribe antibiotics and asthma drugs. Paul et al. [1] determined that each year in the USA, antibiotics are prescribed for nearly $16 \%$ of patients with asthma who present to pediatric ambulatory care settings. According to Knapp et al. [15], visits to an emergency department for moderate to severe asthma result in $29 \%$ of the patients receiving an antibiotic prescription. Coprescribing antibiotics and asthma drugs might be due to asthma severity. However, some studies have shown that, compared to visits to pediatricians, visits to emergency departments by children with respiratory tract infections are less likely to result in a prescription for broad-spectrum antibiotics [16]. Marra et al. [6] also demonstrated that, for pediatric asthmatic patients, rates of prescribing antibiotics were higher in visits to physicians than to specialists. The latter resulted in increased diagnostic certainty of asthma exacerbation and less antibiotic usage. Likewise, our patients were followed prospectively by specialists, which resulted in a decreased use of antibiotics.

Patient asthma education is increasingly being viewed as an important aspect of the ambulatory care setting [17]. Paul et al. [1] demonstrated that asthma education during patient visits is associated with a reduced number of antibiotic prescriptions. The results from this finding suggest other potential benefits for asthma education, as it seems to be associated with more judicious prescribing of antibi- 
otics by providers. Parent-doctor communication during visits is associated with the prescription of antibiotics with the asthma medication. Some parents who believe their children have a disease which should or could be treated with antibiotics may directly request antibiotics [18]. If the patients and their parents are informed and educated about asthma during the visit to the specialist, they are less likely to request antibiotics. This, of course, leads to a general decrease in antibiotic usage [1]. Our patients and their parents were informed and educated at every visit.

The limitation of this study was that for the year prior to the diagnosis of asthma, the patients were evaluated retrospectively. The number of episodes and the frequency of antibiotic usage were obtained from the database and parent reports. The use of a standard classification system for antibiotics may be more reliable. However, in the year after the diagnosis of asthma, patients were regularly followed up prospectively by the specialists and no patients were excluded from the study.

\section{Conclusion}

This study showed that the appropriate diagnosis and treatment of childhood asthma significantly reduce the frequency of antibiotic usage and the number of asthma episodes. Diagnostic certainty about asthma exacerbations, appropriate therapies and regular follow-up contributed to these results as well as follow-up visits to a specialist.

\section{Acknowledgement}

The authors appreciate the contributions and editorial assistance made by S. Delacroix, a native English speaker.

\section{Disclosure Statement}

The authors have no conflicts of interest or funding to disclose.

\section{References}

1 Paul IM, Maselli JH, Hersh AL, et al: Antibiotic prescribing during pediatric ambulatory care visits for asthma. Pediatrics 2011;127: 1014-1021.

-2 Bisgard H, Szefner S: Prevalence of asthmalike symptoms in young children. Pediatr Pulmonol 2007;42:723-728.

- 3 De Boeck K, Vermeulen F, Meyts I, et al: Coprescription of antibiotics and asthma drugs in children. Pediatrics 2011;127:10221026.

4 Hoskin-Parr L, Teyhan L, Blocker A, et al: Antibiotic exposure in the first two years of life and development of asthma and allergic diseases by 7.5 year: a dose-dependent relationship. Pediatr Allergy Immunol 2013;24:762771.

5 Rusconi F, Gagliardi L, Galassi C, et al: Paracetamol and antibiotics in childhood and subsequent development of wheezing/asthma: association or causation? Int J Epidemiol 2011;40:662-667.

6 Marra F, Marra CA, Richardson K, et al: Antibiotic consumption in children prior to diagnosis of asthma. BMC Pulm Med 2011;11: 32.
7 Guilbert TW, Morgan WJ, Krawiec M, et al: The Prevention of Early Asthma in Kids study: design, rationale and methods for the Childhood Asthma Research and Education network. Control Clin Trials 2004;25:286310 .

8 Global Initiative for Asthma: GINA report, global strategy for asthma management and prevention. www.ginasthma.org/Committees.asp? I1 =7\&I2=2 (updated 2011).

9 Khadadah M: The cost of asthma in Kuwait Med Princ Pract 2013;22:87-91.

10 Jansen AG, Sanders EA, Schilder AG, et al: Primary care management of respiratory tract infections in Dutch preschool children. Scand J Prim Health Care 2006;24:231-236.

11 Brand PL, Baraldi E, Bisgaard H, et al: Definition, assessment and treatment of wheezing disorders in preschool children: an evidencebased approach. Eur Respir J 2008;32:10961110.

12 Huang YJ, Nelson CE, Brodie EL, et al: Airway microbiota and bronchial hyper-responsiveness in patients with suboptimally controlled asthma. J Allergy Clin Immunol 2011;127: 372-381.
13 Graham V, Lasserson T, Rowe BH: Antibiotics for acute asthma. Cochrane Database Syst Rev 2001;CD002741.

14 Blasi F, Johnston SL: The role of antibiotics in asthma. Int J Antimicrob Agents 2007;29: 485-493.

15 Knapp JF, Hall M, Sharma V: Benchmarks for the emergency department care of children with asthma, bronchiolitis, and croup. Pediatr Emerg Care 2010;26:364-369.

16 Coco AS, Horst MA, Gambler AS: Trends in broad-spectrum antibiotic prescribing for children with acute otitis media in the United States, 1998-2004. BMC Pediatr 2009;9:41.

17 To T, Guttman A, Lougheed MD, et al: Evidence-based performance indicators of primary care for asthma: a modified RAND Appropriateness Method. Int J Qual Health Care 2010;22:476-485.

18 Strivers T, Mangione-Smith R, Elliott MN, et al: Why do physicians think parents expect antibiotics? What parents report versus what physicians believe. J Fam Pract 2003;52:140148 